\title{
RIQUEZA E SIMILARIDADE FLORÍSTICA DE OITO REMANESCENTES FLORESTAIS NO NORTE DE MINAS GERAIS, BRASIL ${ }^{1}$
}

Rubens Manoel dos Santos², Fábio de Almeida Vieira ${ }^{2}$, Marcílio Fagundes $^{3}$, Yule Roberta Ferreira Nunes ${ }^{3}$ e Eduardo Gusmão ${ }^{4}$

\begin{abstract}
RESUMO - Os objetivos deste estudo foram caracterizar e comparar a composição florística de oito fragmentos de Mata Seca Calcária e Caatinga Arbórea localizados no norte do Estado de Minas Gerais. O levantamento das espécies arbóreas foi realizado através de visitas periódicas aos fragmentos para coleta de material botânico. Nos oito fragmentos foram encontradas 114 espécies arbóreas pertencentes a 40 famílias botânicas. Dentre as 89 espécies encontradas nos fragmentos de Mata Seca, 37 (42\%) foram peculiares a essa formação. No entanto, das 75 espécies amostradas nos remanescentes de Caatinga Arbórea, 25 (33\%) ocorreram apenas nesses fragmentos. A análise de agrupamento sugere que a composição florística dos fragmentos é afetada pela fitofisionomia e pelas condições bioecológicas locais a que estes fragmentos estão submetidos. Além disso, a relação observada entre a área dos fragmentos e o número de espécies arbóreas, evidenciou que área é um bom indicador da riqueza de espécies arbóreas nos fragmentos florestais. Portanto, os resultados deste estudo indicaram que fatores históricos (fitofisionomia) e ecológicos (área dos fragmentos e ocorrência de perturbações antrópicas) constituem importantes elementos caracterizadores da composição e riqueza de espécies em fragmentos florestais do norte de Minas Gerais.
\end{abstract}

Palavras-chave: Florística, Fragmentação Florestal, Floresta Estacional Decidual, Mata Seca Calcária e Caatinga Arbórea.

\section{FLORISTIC RICHNESS AND SIMILARITY OF EIGHT FOREST REMNANTS IN THE NORTH OF MINAS GERAIS STATE, BRAZIL}

\begin{abstract}
The objective of this study was to characterize and compare the floristic composition of eight fragments of Calcareous Dry Forest and Arboreal Caatinga in the north of Minas Gerais State, Brazil. The survey of the tree species was performed by periodic visits to the fragments. We found 114 tree species belonging to 40 botanical families in the eight fragments. Of a total of 89 species found in the Calcareous Dry Forest fragments, 37 (42\%) were typical of this formation. On the other hand, from the 75 species sampled in the Arboreal Caatinga remnants, 25 species (33\%) occurred only in these fragments. The cluster analysis suggested that the floristic composition of the fragments was affected by the phytophysiognomy and by local bioecological conditions. The observed relationship between remnant area and tree species richness suggests that area was a good indicator of species richness in the forest fragments. The results of this study indicate that both historical (phytophysiognomy) and ecological (e.g., remnant area and occurrence of human disturbance) factors constitute important elements that determine the composition and richness of tree species of forest fragments in the north of Minas Gerais State.
\end{abstract}

Keywords: floristic, fragmentation, Deciduous Dry Forests, Calcareous Dry Forests, and Arboreal Caatinga.

\footnotetext{
${ }^{1}$ Recebido em 14.03.2006 e aceito para publicação em 13.09.2006.

${ }^{2}$ Programa de Pós-Graduação em Engenharia Florestal da Universidade Federal de Lavras, 37200-000, Lavras-MG.

${ }^{3}$ Departamento de Biologia Geral da Universidade Estadual de Montes Claros, 39401-089 Montes Claros-MG.

E-mail:<yule.nunes@unimontes.br>.

${ }^{4}$ Programa de Pós-Graduação em Fisiologia Vegetal da Universidade Federal de Viçosa, 36570-000 Viçosa-MG.
} 


\section{INTRODUÇÃO}

O Estado de Minas Gerais possui diversificadas formações vegetais, resultantes provavelmente da sua ampla variação nas condições geológicas, topográficas e climáticas (MELLO-BARRETO, 1942). Especificamente, a região norte do Estado se caracteriza por apresentar vegetação que expressa uma condição de sobrevivência ligada à deficiência hídrica, adaptada a um clima severo e com baixa precipitação anual distribuída em um curto período do ano (FERNANDES, 2002). Essa região pertence ao domínio das Caatinga em sua parte norte e nordeste, com transição para o Cerrado, ao sul e a oeste (BRANDÃO, 1994). Assim, duas fisionomias florísticas bem distintas e características têm seus limites na região norte do Estado de Minas Gerais, sendo uma representada pela área seca da Caatinga e a outra pelo Cerrado (MARTIUS, 1958).

Áreas de transição entre os Domínios da Caatinga e do Cerrado podem ser perfeitamente observadas nessa região, dando origem a fitofisionomias bem diferenciadas, principalmente no que se refere a composição de espécies e à abundância e porte dos indivíduos. Dentre as fitofisionomias florestais observadas nesse ecótone, destacam-se a Mata Seca Calcária e a Caatinga Arbórea (BRANDÃO, 1994). Segundo Fernandes (1998), a Caatinga Arbórea é extremamente rara, ocorrendo sobretudo no norte de Minas Gerais. Entre as espécies mais representativas dessa formação vegetal, destacamse Auxemma oncolalyx, A. glazioviana, Caesalpinia bracteosa, C. microphylla, C. ferrea, Cnidosculus phyllacanthus, Fraunhofera multiflora, Mimosa caesalpiniifolia, Myracrodruon urundeuva e Patagonula bahiana. No entanto, a Mata Seca Calcária ocorre em áreas de afloramento calcário no Cerrado e apresenta espécies arbóreas com maiores tamanhos (área basal e altura) que a vegetação de Cerrado nas suas distintas fisionomias (NASCIMENTO et al., 2004). Além disso, o ritmo estacional, marcado pelo elevado grau de deciduidade foliar durante a seca, constitui outro aspecto caracterizador dessa formação (PEDRALLI, 1997). No norte de Minas Gerais, as espécies mais comuns da Mata Seca Calcária são Anadenanthera macrocarpa, Astronium fraxinifolium, Ceiba glaziovii, Guazuma ulmifolia, Hortia arborea, Machaerium acutifolium, Melanoxylum braunia, Platymiscium floribundum, Sapindus saponaria, Spondias mombim, Tabebuia serrratifolia, Talisia esculenta, Triplaris gardneriana e Zollernia ilicifolia (BRANDÃO, 1994; PEDRALLI, 1997).

R. Árvore, Viçosa-MG, v.31, n.1, p.135-144, 2007
Apesar da particularidade dessas formações, elas são pouco estudadas, e suas áreas originais têm sido reduzidas (OLIVEIRA-FILHO e MACHADO, 1993). Esse processo de desmatamento resulta na formação de fragmentos florestais esparsos, que constituem os últimos refúgios para muitas espécies vegetais e animais (ESPÍRITO-SANTO et al., 2002). De fato, o processo de fragmentação afeta a organização das comunidades naturais, especialmente porque reduz a área de vida das espécies e altera as condições climáticas locais (BIERREGAARD e DALE, 1996).

Atualmente, boa parte da biodiversidade encontrase concentrada em fragmentos isolados de ambientes naturais (VIANA e PINHEIRO, 1998). Além disso, a capacidade desses sistemas de conservar ou não a biodiversidade regional e resistir à prolongada intervenção humana é assunto para estudos particularmente relevantes (KELLMAN et al., 1996). Portanto, a possibilidade de que sistemas de pequenos fragmentos representem a maior porcentagem dos refúgios naturais em uma paisagem tropical faz da possibilidade de previsão das condições futuras nesses ambientes um assunto de extrema importância para a biologia da conservação. Assim, este trabalho foi desenvolvido com o objetivo de conhecer a composição florística das comunidades arbóreas de oito fragmentos florestais localizados no norte do Estado de Minas Gerais. Além disso, avaliou-se a similaridade florística entre esses fragmentos em razão da localização geográfica e da fitofisionomia local. Finalmente, caracterizouse o efeito do tamanho dos fragmentos na riqueza de espécies arbóreas desses remanescentes florestais.

\section{MATERIAL E MÉTODOS}

Este estudo foi realizado em oito fragmentos florestais, pertencentes a quatro municípios distintos (Juvenília, Montalvânia, Juramento e Montes Claros), localizados na região norte do Estado de Minas Gerais. Esses fragmentos, denominados Mata da Serra (MS), Volta da Serra (VS), Vale Verde (VV), Pedra Preta (PP), Lapinha (La), Escola Caio Martins (ECM), Parque da Sapucaia (PS) e COPASA (CO), ocorrem entre as coordenadas $14^{\circ} 15^{\prime}$ '93' a 16 46'20' latitude sul e 4339'56" a 4430' $58^{\prime \prime}$ longitude oeste e apresentam áreas variando de 8 a 2.337 ha. Esses remanescentes foram classificados fisionomicamente como Caatinga Arbórea ou Mata Seca Calcária (BRANDÃO, 1994), caracterizados como Florestas Estacionais Deciduais (VELOSO et al., 1991), 
sendo ainda observadas as diferentes fisionomias circunvizinhas e a altura dominante do dossel desses ambientes. (Quadro 1).

O inventário florístico foi realizado através de caminhadas aleatórias por todos os fragmentos estudados durante o período de setembro de 2001 a maio de 2003. Durante esse período foram realizadas quatro visitas a cada fragmento, utilizando-se, desse modo, o mesmo esforço amostral. Nessas visitas, foram realizadas incursões ao interior dos fragmentos, através de caminhadas aleatórias, que variavam de quatro a cinco horas, para caracterizar a flora local e coletar material botânico. Neste estudo, foram amostradas todas as diferentes espécies arbóreas visualizadas, com DAP (diâmetro à altura do peito $=1,30 \mathrm{~m}$ do solo) $\geq$ a $5 \mathrm{~cm}$. O material vegetal coletado foi tratado segundo as técnicas convencionais de herborização e depositado no Herbário Montes Claros (HMC), da Universidade Estadual de Montes Claros (UNIMONTES). A identificação do material botânico coletado foi feita através de consultas a especialistas, uso de literatura especializada e comparações com as exsicatas do HMC. O sistema de classificação das espécies adotado foi o Angiosperm Phylogeny Group II (APG II, 2003).

A similaridade florística entre os fragmentos foi determinada, através da análise de agrupamento, sobre a matriz básica de presença/ausência das espécies arbóreas amostradas. Utilizou-se a distância euclidiana como medida de similaridade florística entre os fragmentos e a ligação simples como medida de ligação (LUDWIG e REYNOLDS, 1986). O padrão de agrupamento encontrado apresentou pequena variação quando outros coeficientes de medida de similaridade foram usados (e.g. distância euclidiana média, distância média absoluta, distância euclidiana quadrática, distância relativa absoluta), indicando que o padrão é consistente. Finalmente, para avaliar o efeito da distância entre os fragmentos na similaridade florística procedeu-se à análise de regressão linear (ZAR, 1996), usando a distância geográfica linear entre os fragmentos como variável independente e a similaridade florística (obtida na análise de agrupamento) como variável dependente. $\mathrm{O}$ efeito do tamanho dos fragmentos na riqueza de espécies foi avaliado através de regressão linear (ZAR, 1996), utilizando-se o número de espécies por fragmento como variável dependente e a área do fragmento como variável independente. Nesse caso, as áreas dos diferentes fragmentos foram transformadas em escala logarítmica, para melhor linearização dos dados.

\section{RESULTADOS E DISCUSSÃO}

Nos oito fragmentos estudados foram amostradas 114 espécies arbóreas pertencentes a 40 famílias botânicas (Quadro 2). Seis espécies se destacaram devido à alta freqüência, ocorrendo em todos os fragmentos: Anadenanthera colubrina, Aspidosperma pyrifolium, Myracrodruon urundeuva, Schinopsis brasiliensis, Tabebuia roseo-alba e Tabebuia cf. chrysotricha. Entretanto, um expressivo número de espécies (33\%) apresentou baixa frequiência, ocorrendo em um único fragmento. Além disso, dentre as 89 espécies encontradas nos fragmentos de Mata Seca, 37 (42\%) foram exclusivas dessa formação. Entranto, das 75 espécies amostradas nos remanescentes de Caatinga Arbórea, 25 (33\%) foram de ocorrência apenas nesses fragmentos. Portanto, apenas $46 \%$ das espécies ocorreram nas duas formações, salientando a ampla variação florística entre os fragmentos de Mata Seca Calcária e de Caatinga Arbórea (Quadro 2). Esses resultados ressaltam a alta substituição de espécies entre os fragmentos, indicando que, apesar da semelhança fisionômica entre as áreas, pode haver variação na composição de espécies entre localidades específicas. De fato, Cavanilesia arborea, Cnidoscolus pubescens, Pseudobombax simplicifolium, Sterculia striata e Syagrus oleracea mostraram-se bons representantes da Caatinga Arbórea, enquanto Astronium fraxinifolium, Bauhinia sp.1, Genipa americana e Heteropteryx byrsonimifolia foram específicos dos fragmentos da Mata Seca Calcária. Contudo, a escassez de estudos florísticos nessas formações florestais, especialmente no norte de Minas Gerais, ainda não permite inferências mais conclusivas sobre a distribuição das espécies entre essas formações.

O padrão de similaridade florística resultante da análise de agrupamentos evidencia que a composição florística dos fragmentos de Mata Seca Calcária difere acentuadamente dos fragmentos de Caatinga arbórea. Além disso, a similaridade florística observada indicou que a composição de espécies dos fragmentos é amplamente afetada pela formação florestal a que esses fragmentos pertencem (Figura 1). De fato, os fragmentos da COPASA e do Parque da Sapucaia, pertencentes ao Domínio do Cerrado, formaram um subgrupo distinto dos demais fragmentos que ocorrem na Caatinga (Quadro 1). Do mesmo modo, a análise de agrupamentos também

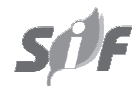

R. Árvore, Viçosa-MG, v.31, n.1, p.135-144, 2007 
apontou que os fragmentos de Caatinga Arbórea podem ser subdivididos em dois subgrupos: (I) Escola Caio Martins, Pedra Preta e Lapinha e (II) Vale Verde, Volta da Serra e Mata da Serra (Figura 1). Os fragmentos do subgrupo I encontram-se mais preservados, fazendo limites com outras formações menos antropizadas, enquanto os fragmentos do subgrupo II são mais antropizados e ocorrem próximos a afloramentos calcários. Assim, é provável que essa subdivisão dos fragmentos da Caatinga arbórea se encontre associada a aspectos ecológicos locais, como as características edáficas e, ou, históricas de perturbações. Nesse sentido, vários estudos têm salientado a importância de fatores ambientais na composição de espécies de remanescentes florestais (OLIVEIRA-FILHOetal., 1998; van den BERGe OLIVEIRA FILHO, 1999; SOUZA et al., 2003).

De fato, diversos fatores abióticos como o clima (WILLIANS-LINERA et al., 1998), a qualidade do solo (e.g. OLIVEIRA-FILHO et al., 1998; ESPÍRITO-SANTO et al., 2002), a ocorrência de cursos d'água (MEYER et al., 2004) e distúrbios (NUNES et al., 2003; RIBAS et al., 2003) podem afetar a distribuição e abundância das espécies arbóreas, dentro de "habitats" específicos, agindo diretamente na dinâmica das populações (VIANA e PINHEIRO, 1998).

Quadro 1 - Informações gerais sobre os oito fragmentos inventariados na região norte de Minas Gerais: Mata da Serra (MS), Volta da Serra (VS), Vale Verde (VV), Pedra Preta (PP), Lapinha (La), Escola Caio Martins (ECM), Parque da Sapucaia (PS), COPASA (CO), Caatinga Arbórea = CA, Mata Seca Calcária = MS, Pastagem = P, Capoeira $=\mathrm{CP}$, Afloramento de Calcário $=\mathrm{AC}$, Cerrado $=\mathrm{CE}$ e Mata Ciliar $=\mathrm{MC}$

Table 1 - General information of the eight fragments studied in the north of Minas Gerais State: Mata da Serra (MS), Volta da Serra (VS), Vale Verde (VV), Pedra Preta (PP), Lapinha (La), Escola Caio Martins (ECM), Parque da Sapucaia (PS), COPASA (CO). Arboreal Caatinga $=$ CA, Calcareus Dry Forest $=M S$, Pasture $=$ P, Early stage of succession $=C P$, Calcareus soils $=A C$, Cerrado $=C E$ and Gallery Forest $=M C$

\begin{tabular}{|c|c|c|c|c|c|c|c|}
\hline Código & Município & $\begin{array}{c}\text { Área } \\
\text { (ha) }\end{array}$ & $\begin{array}{c}\text { Latitude } \\
\text { (S) }\end{array}$ & $\begin{array}{c}\text { Longitude } \\
\text { (W) }\end{array}$ & $\begin{array}{c}\text { Altura do } \\
\text { dossel }(\mathrm{m})\end{array}$ & Fisionomia & Limites \\
\hline MS & Juvenília & 60 & $14^{\circ} 28^{\prime} 22^{\prime \prime}$ & $44^{\circ} 11^{\prime} 30^{\prime \prime}$ & 22 & $\mathrm{CA}$ & $\mathrm{P}, \mathrm{CP}, \mathrm{AC}$ \\
\hline VS & Juvenília & 45 & $14^{\circ} 26^{\prime} 04^{\prime \prime}$ & $44^{\circ} 10^{\prime} 67^{\prime}$, & 20 & $\mathrm{CA}$ & $\mathrm{P}, \mathrm{AC}$ \\
\hline W & Juvenília & 100 & $14^{\circ} 24^{\prime} 88^{\prime \prime}$ & $44^{\circ} 09^{\prime} 79^{\prime \prime}$ & 14 & $\mathrm{CA}$ & $\mathrm{AC}, \mathrm{CP}, \mathrm{P}$ \\
\hline P P & Montalvânia & 80 & $14^{\circ} 27^{\prime} 68^{\prime \prime}$ & $44^{\circ} 30^{\prime} 58^{\prime \prime}$ & 10 & $\mathrm{CA}$ & $\mathrm{CE}, \mathrm{CP}, \mathrm{P}$ \\
\hline $\mathrm{La}$ & Juvenília & 150 & $14^{\circ} 29^{\prime} 26^{\prime \prime}$ & $44^{\circ} 11^{\prime} 03^{\prime \prime}$ & 22 & $\mathrm{CA}$ & $\mathrm{CP}, \mathrm{CR}, \mathrm{AC}, \mathrm{P}, \mathrm{MC}$ \\
\hline $\mathrm{ECM}$ & Juvenília & 8 & $14^{\circ} 15^{\prime} 93^{\prime \prime}$ & $44^{\circ} 06^{\prime} 42^{\prime \prime}$ & 15 & $\mathrm{CA}$ & $\mathrm{CE}, \mathrm{MC}, \mathrm{P}, \mathrm{CP}$ \\
\hline PS & Montes Claros & 30 & $16^{\circ} 44^{\prime} 57^{\prime \prime}$ & $43^{\circ} 54^{\prime} 13^{\prime \prime}$ & 10 & MS & $\mathrm{MC}$ \\
\hline $\mathrm{CO}$ & Juramento & 2337 & $16^{\circ} 46^{\prime} 20^{\prime \prime}$ & $43^{\circ} 39^{\prime} 56^{\prime \prime}$ & 10 & MS & $\mathrm{MC}, \mathrm{CE}, \mathrm{CP}$ \\
\hline
\end{tabular}

Quadro 2 - Espécies arbóreas encontradas em oito fragmentos de Caatinga Arbórea e Mata Seca Calcária no norte de Minas Gerais, Brasil (MS: Mata da Serra, VS: Volta da Serra, VV: Vale Verde, PP: Pedra Preta, La: Lapinha, ECM: Escola Caio Martins, PS: Parque da Sapucaia, CO: COPASA, X: presença da espécie no fragmento)

Table 2 - Arboreal species survey in the eight fragments of Arboreal Caatinga and Calcareus Dry Forest in the north of Minas Gerais State, Brazil (MS: Mata da Serra, VS: Volta da Serra, VV: Vale Verde, PP: Pedra Preta, La: Lapinha, ECM: Escola Caio Martins, PS: Parque da Sapucaia, CO: COPASA, X: presence of the specie in the fragment)

\begin{tabular}{|c|c|c|c|c|c|c|c|c|}
\hline Espécie & MS & VS & W & $\mathrm{PP}$ & $\mathrm{La}$ & ECM & PS & $\mathrm{CO}$ \\
\hline \multicolumn{9}{|l|}{ ANACARDIACEAE } \\
\hline Astronium fraxinifolium Schott & & & & & & & $X$ & $\mathrm{X}$ \\
\hline Lithraea molleoides (Vell.) Engl. & & & & & & & $\mathrm{X}$ & \\
\hline Myracrodruon urundeuva Fr. All. & $\mathrm{X}$ & $\mathrm{X}$ & $\mathrm{X}$ & $\mathrm{X}$ & $\mathrm{X}$ & $\mathrm{X}$ & $\mathrm{X}$ & $\mathrm{X}$ \\
\hline Schinopsis brasiliensis Engl. & $\mathrm{X}$ & $\mathrm{X}$ & $\mathrm{X}$ & $\mathrm{X}$ & $\mathrm{X}$ & $\mathrm{X}$ & $\mathrm{X}$ & $\mathrm{X}$ \\
\hline Spondias tuberosa Arruda & $X$ & $\mathrm{X}$ & $\mathrm{X}$ & & & & & \\
\hline \multicolumn{9}{|l|}{ ANNONACEAE } \\
\hline Annona crassiflora Mart. & & & & & & & & $\mathrm{X}$ \\
\hline Rollinia silvatica (St. Hil.) Mart. & $X$ & & & $\mathrm{X}$ & & & & $\mathrm{X}$ \\
\hline $\begin{array}{l}\text { AQUIFOLIACEAE } \\
\text { Ilex brevicuspis Reissek }\end{array}$ & $X$ & & $X$ & & $X$ & & $X$ & \\
\hline
\end{tabular}

R. Árvore, Viçosa-MG, v.31, n.1, p.135-144, 2007 
Quadro 2 - Cont

Table 2-Cont.

\begin{tabular}{|c|c|c|c|c|c|c|c|c|}
\hline Espécie & MS & $\mathrm{VS}$ & $\mathrm{WV}$ & $\mathrm{PP}$ & $\mathrm{La}$ & ECM & PS & $\mathrm{CO}$ \\
\hline $\begin{array}{l}\text { ARALIACEAE } \\
\text { Sciadodendron excelsum } \text { Griseb. }\end{array}$ & $X$ & & $X$ & & $\mathrm{X}$ & & $X$ & $\mathrm{X}$ \\
\hline $\begin{array}{l}\text { ARECACEAE } \\
\text { Acrocomia aculeata (Jacq.) Lodd. }\end{array}$ & & & & & & & $X$ & \\
\hline $\begin{array}{l}\text { Syagrus oleracea (Mart.) Becc. } \\
\text { APOCYNACEAE }\end{array}$ & $X$ & $X$ & $X$ & & $X$ & $X$ & & \\
\hline $\begin{array}{l}\text { Aspidosperma pyrifolium Mart. } \\
\text { Aspidosperma } \mathrm{sp} .\end{array}$ & $X$ & $\mathrm{X}$ & $\mathrm{X}$ & $X$ & $\mathrm{X}$ & $X$ & $\begin{array}{l}X \\
X\end{array}$ & $\mathrm{X}$ \\
\hline
\end{tabular}

\section{BIGNONIACEAE}

Cybistax antisyphilitica Mart.

Jacaranda brasiliana (Lam.) Pers.

Tabebuia roseo-alba (Ridl.) Sand.

Tabebuia cf. chrysotricha (Mart. ex DC.) Standl.

Zeyheria tuberculosa Bur.

BURSERACEAE

Commiphora leptophloeos (Mart.) Gillett

\section{CACTACEAE}

Cereus jamacaru Hort. Vindob. ex Salm-Dyck.

Pereskia sp.

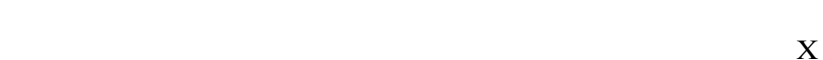

\section{CECROPIACEAE}

Cecropia pachystachya Trec.

CELASTRACEAE

Maytenus sp.

\section{COMBRETACEAE}

Combretum leprosum Mart.

Terminalia argentea Mart.

Terminalia brasiliensis $\mathrm{Camb}$

\section{CLUSIACEAE}

Vismia sp.

DILLENIACEAE

Curatella americana $\mathrm{L}$.

EBENACEAE

Diospyros brasiliensis Mart.

$\begin{array}{cccccccc}\underline{\mathrm{X}} & \underline{\mathrm{X}} & \underline{\mathrm{X}} & \underline{\mathrm{X}} & \underline{\mathrm{X}} & \underline{\mathrm{X}} & \underline{\mathrm{X}} & \underline{\mathrm{X}} \\ \mathrm{X} & \mathrm{X} & \mathrm{X} & \mathrm{X} & \mathrm{X} & \mathrm{X} & & \mathrm{X} \\ \mathrm{X} & \mathrm{X} & \mathrm{X} & \mathrm{X} & & & & \\ \mathrm{X} & \mathrm{X} & \mathrm{X} & & & & \\ \mathrm{X} & \mathrm{X} & \mathrm{X} & & \mathrm{X} & & & \end{array}$

ERYTHROXYLACEAE

Erythroxylum deciduum St. Hil.

EUPHORBIACEAE

Cnidoscolus pubescens (Pax.) Pax. \& K. Hoffm.

Croton urucurana Baill.

Croton sp.

Jatropha sp.

Manihot sp.

Sapium sp.

FABACEAE CAESALPINIOIDEAE

Apuleia leiocarpa (Vog.) Macbr.

Bauhinia forficata Link

Bauhinia sp.1

Bauhinia sp.2

Caesalpinia microphylla Mart.

Copaifera langsdorffii Desf.

Deguelia costata (Benth.) Az. Tozzi

Goniorrhachis marginata Taub.

$x$

Hymenaea stignocarpa Mart. ex Hayne

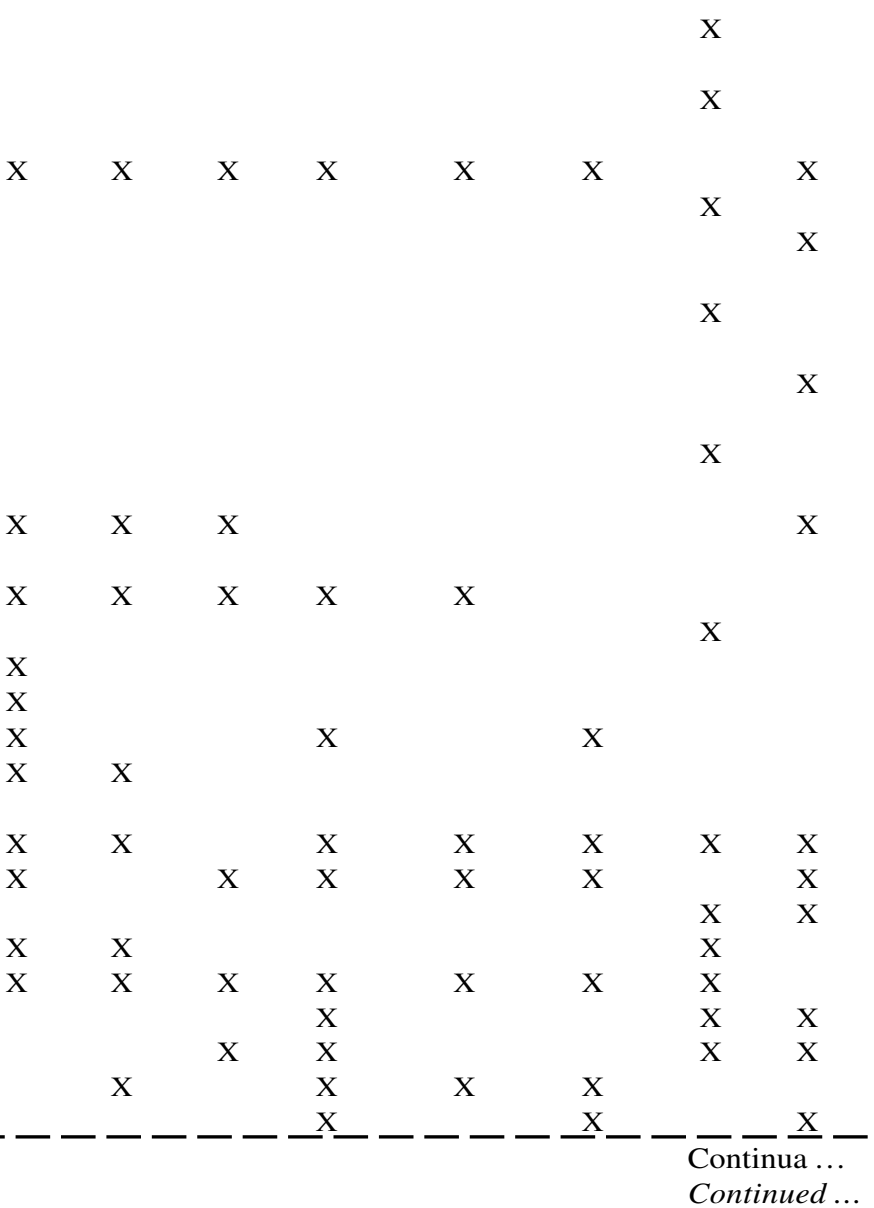


Quadro 2 - Cont

Table 2-Cont.

\begin{tabular}{|c|c|c|c|c|c|c|c|c|}
\hline Espécie & MS & VS & $\mathrm{V}$ & $\mathrm{PP}$ & $\mathrm{La}$ & ECM & PS & $\mathrm{CO}$ \\
\hline \multicolumn{9}{|l|}{ FABACEAE CAESALPINIOIDEAE } \\
\hline Machaerium aculeatum Raddi & $\mathrm{X}$ & & $\mathrm{X}$ & & & $\mathrm{X}$ & $\mathrm{X}$ & $\mathrm{X}$ \\
\hline Machaerium brasiliense Vogel & $\mathrm{X}$ & $\mathrm{X}$ & $\mathrm{X}$ & $\mathrm{X}$ & $\mathrm{X}$ & $\mathrm{X}$ & & $\mathrm{X}$ \\
\hline Machaerium hirtum (Vell.) Stellf. & & & & & $\mathrm{X}$ & & $\mathrm{X}$ & \\
\hline Machaerium opacum Vog. & & & & & $\mathrm{X}$ & & $\mathrm{X}$ & $\mathrm{X}$ \\
\hline Machaerium scleroxylon Tul. & & & & $\mathrm{X}$ & & $\mathrm{X}$ & $\mathrm{X}$ & $\mathrm{X}$ \\
\hline Machaerium sp. & & & & $\mathrm{X}$ & & & & \\
\hline Peltophorum dubium (Spreng.) Taub. & $\mathrm{X}$ & $\mathrm{X}$ & & & & $\mathrm{X}$ & & $\mathrm{X}$ \\
\hline Platypodium elegans Vogel & & & & & & & & $\mathrm{X}$ \\
\hline Platymiscium blanchetii Benth. & & & $\mathrm{X}$ & & & & & $\mathrm{X}$ \\
\hline Platymiscium floribundum Vog. & & & $\mathrm{X}$ & & & $\mathrm{X}$ & $\mathrm{X}$ & $\mathrm{X}$ \\
\hline Pterogyne nitens Tul. & $\mathrm{X}$ & $\mathrm{X}$ & & & $\mathrm{X}$ & $\mathrm{X}$ & $\mathrm{X}$ & $\mathrm{X}$ \\
\hline Senna spectabilis (DC.) Irwin et Barn. & $\mathrm{X}$ & $\mathrm{X}$ & $\mathrm{X}$ & & $\mathrm{X}$ & & $\mathrm{X}$ & \\
\hline \multicolumn{9}{|l|}{ FABACEAE FABOIDEAE } \\
\hline Acosmiun dasycarpum (Vog.) Yakovl. & & & & & & & & $\mathrm{X}$ \\
\hline Erythrina mulungu Mart. & $\mathrm{X}$ & & & & & & & \\
\hline \multicolumn{9}{|l|}{ FABACEAE MIMOSOIDEAE } \\
\hline Acacia bahiensis Benth. & $\mathrm{X}$ & $\mathrm{X}$ & $\mathrm{X}$ & $\mathrm{X}$ & & & $\mathrm{X}$ & $\mathrm{X}$ \\
\hline Albizia blanchettii (Benth.) & & & & & & & & $\mathrm{X}$ \\
\hline Albizia hassleri (Chodat) Burr. & & & & & & $\mathrm{X}$ & & $\mathrm{X}$ \\
\hline Albizia sp. & $\mathrm{X}$ & $\mathrm{X}$ & $\mathrm{X}$ & & $\mathrm{X}$ & $\mathrm{X}$ & & $\mathrm{X}$ \\
\hline Anadenanthera colubrina (Vell.) Brenan & $\mathrm{X}$ & $\mathrm{X}$ & $\mathrm{X}$ & $\mathrm{X}$ & $\mathrm{X}$ & $\mathrm{X}$ & $\mathrm{X}$ & $\mathrm{X}$ \\
\hline Chloroleucon tortum (Mart.) Pittier & $\mathrm{X}$ & $\mathrm{X}$ & $\mathrm{X}$ & $\mathrm{X}$ & $\mathrm{X}$ & $\mathrm{X}$ & & $\mathrm{X}$ \\
\hline Inga sessilis (Vell.) Mart. & & & & & & & & $\mathrm{X}$ \\
\hline Mimosa tenuiflora (Willd.) Poiret & $\mathrm{X}$ & & $\mathrm{X}$ & $\mathrm{X}$ & & $\mathrm{X}$ & & \\
\hline Piptadenia viridiflora (Kunth) Benth. & $\mathrm{X}$ & $\mathrm{X}$ & $\mathrm{X}$ & & & $\mathrm{X}$ & $\mathrm{X}$ & $\mathrm{X}$ \\
\hline LAMIACEAE & & & & & & & & \\
\hline Vitex cymosa Bert. & $\mathrm{X}$ & & & & & & & $\mathrm{X}$ \\
\hline \multicolumn{9}{|l|}{ LAURACEAE } \\
\hline Nectandra sp. & & & & & & & $\mathrm{X}$ & \\
\hline \multicolumn{9}{|l|}{ LECYTHIDACEAE } \\
\hline Cariniana estrellensis (Raddi) Kuntze & & & & & & & $\mathrm{X}$ & \\
\hline \multicolumn{9}{|l|}{ MALPIGHIACEAE } \\
\hline Byrsonima verbacifolia (L.) Rich & & & & & & & & $\mathrm{X}$ \\
\hline Heteropterys byrsonimifolia A. Juss & & & & & & & $\mathrm{X}$ & $\mathrm{X}$ \\
\hline \multicolumn{9}{|l|}{ MALVACEAE } \\
\hline Cavanillesia arborea K. Schum. & $\mathrm{X}$ & $\mathrm{X}$ & $\mathrm{X}$ & $\mathrm{X}$ & $\mathrm{X}$ & & & \\
\hline Eriotheca pubescens Schott et Endl. & & & & & & & & $\mathrm{X}$ \\
\hline Ceiba speciosa St. Hil. & & & $\mathrm{X}$ & & & & & \\
\hline Guazuma ulmifolia Lam. & & & & & $\mathrm{X}$ & $\mathrm{X}$ & $\mathrm{X}$ & $\mathrm{X}$ \\
\hline Luehea divaricata Mart. & $\mathrm{X}$ & & & $\mathrm{X}$ & & & $\mathrm{X}$ & $\mathrm{X}$ \\
\hline Pseudobombax simplicifolium A. Robyns & $\mathrm{X}$ & $\mathrm{X}$ & $\mathrm{X}$ & & $\mathrm{X}$ & $\mathrm{X}$ & & \\
\hline Sterculia striata St. Hil. et Naud. & $\mathrm{X}$ & $\mathrm{X}$ & $\mathrm{X}$ & $\mathrm{X}$ & & $\mathrm{X}$ & & \\
\hline \multicolumn{9}{|l|}{ MELIACEAE } \\
\hline Cedrela fissilis Vell. & $\mathrm{X}$ & $\mathrm{X}$ & & $\mathrm{X}$ & & & $\mathrm{X}$ & $\mathrm{X}$ \\
\hline Trichilia hirta $\mathrm{L}$. & $\mathrm{X}$ & & $\mathrm{X}$ & $\mathrm{X}$ & $\mathrm{X}$ & $\mathrm{X}$ & $\mathrm{X}$ & \\
\hline $\begin{array}{l}\text { MORACEAE } \\
\text { Brosimum gaudichaudii Trec. }\end{array}$ & & & & & & & & $\mathrm{X}$ \\
\hline
\end{tabular}

Ficus gameleira Standl.

Ficus sp.

Maclura tinctoria D. Don ex Steud.

$\mathrm{X}$

$\mathrm{X}$

$\mathrm{X} \quad \mathrm{X} \quad \mathrm{X} \quad \mathrm{X} \quad \mathrm{X}$ 
Quadro 2-Cont.

Table 2-Cont.

\begin{tabular}{|c|c|c|c|c|c|c|c|c|}
\hline Espécie & MS & $\mathrm{VS}$ & $\mathrm{V}$ & $\mathrm{PP}$ & $\mathrm{La}$ & ECM & PS & $\mathrm{CO}$ \\
\hline \multicolumn{9}{|l|}{ MYRTACEAE } \\
\hline Eugenia cf. florida DC. & $\mathrm{X}$ & & $\mathrm{X}$ & $\mathrm{X}$ & $\mathrm{X}$ & $\mathrm{X}$ & $\mathrm{X}$ & $\mathrm{X}$ \\
\hline Eugenia desinterica DC. & & & & & & & & $\mathrm{X}$ \\
\hline Eugenia florida DC. & & & & & & & $\mathrm{X}$ & \\
\hline Myrciaria floribunda & & & & & & & $\mathrm{X}$ & \\
\hline Psidium sp. & & & & & & $\mathrm{X}$ & & $\mathrm{X}$ \\
\hline \multicolumn{9}{|l|}{ NYCTAGINACEAE } \\
\hline Bougainvillea praecox Griseb. & $\mathrm{x}$ & & & $\mathrm{X}$ & $\mathrm{X}$ & & & $\mathrm{X}$ \\
\hline Guapira opposita Vell. & $\mathrm{X}$ & & & & & & $\mathrm{X}$ & \\
\hline \multicolumn{9}{|l|}{ PIPERACEAE } \\
\hline Piper sp. & & & & & & & $\mathrm{X}$ & \\
\hline \multicolumn{9}{|l|}{ POLYGONACEAE } \\
\hline Coccoloba sp. & $\mathrm{X}$ & $\mathrm{X}$ & $\mathrm{X}$ & & & & & \\
\hline Triplaris gardneriana Wedd. & & & & & $\mathrm{X}$ & & & $\mathrm{X}$ \\
\hline \multicolumn{9}{|l|}{ PROTEACEAE } \\
\hline Roupala brasiliensis Klotz. & & & & & & & & $\mathrm{X}$ \\
\hline \multicolumn{9}{|l|}{ RHAMNACEAE } \\
\hline Rhamnidium elaeocarpum Reiss. & & & & & $\mathrm{X}$ & & $\mathrm{X}$ & $\mathrm{X}$ \\
\hline \multicolumn{9}{|l|}{ RUBIACEAE } \\
\hline Alibertia concolor (Cham.) K. Schum & & & & & & & $\mathrm{X}$ & \\
\hline Genipa americana $\mathrm{L}$. & & & & & & & $\mathrm{X}$ & $X$ \\
\hline Randia armata (Sw.) DC. & $\mathrm{X}$ & $\mathrm{X}$ & $\mathrm{X}$ & $\mathrm{X}$ & $\mathrm{X}$ & & & $X$ \\
\hline Coutarea hexandra (Jacq.) K. Schum. & $\mathrm{X}$ & & & $\mathrm{X}$ & $\mathrm{X}$ & & & \\
\hline \multicolumn{9}{|l|}{ RUTACEAE } \\
\hline Esenbeckia sp. & & & & & & & $\mathrm{X}$ & \\
\hline Zanthoxylum riedelianum Engl. & $\mathrm{X}$ & & $\mathrm{X}$ & & & & $\mathrm{X}$ & $\mathrm{X}$ \\
\hline \multicolumn{9}{|l|}{ SALICACEAE } \\
\hline \multicolumn{8}{|l|}{ SAPINDACEAE } & \\
\hline $\begin{array}{l}\text { SAPINDACEAE } \\
\text { Allophyllus edulis (St. Hil.) Radik. }\end{array}$ & $\mathrm{X}$ & & $\mathrm{X}$ & & $\mathrm{X}$ & & & \\
\hline Dilodendron bipinnatum Radik. & $\mathrm{X}$ & & & & & & $\mathrm{X}$ & $\mathrm{X}$ \\
\hline Talisia esculenta (St. Hil.) Radlk. & & & & & $\mathrm{X}$ & $\mathrm{X}$ & $\mathrm{X}$ & $\mathrm{X}$ \\
\hline \multicolumn{9}{|l|}{ SOLANACEAE } \\
\hline Solanum lycocarpum St. Hil. & & & & & & & & $\mathrm{X}$ \\
\hline Solanum sp. 1 & $\mathrm{X}$ & & & & & & & \\
\hline Solanum sp.2 & $\mathrm{X}$ & $\mathrm{X}$ & & & & & & \\
\hline \multicolumn{9}{|l|}{ ULMACEAE } \\
\hline Celtis iguanea (Jacq.) Sarg. & $\mathrm{X}$ & $\mathrm{X}$ & & $\mathrm{x}$ & $\mathrm{X}$ & $\mathrm{X}$ & $\mathrm{X}$ & $\mathrm{X}$ \\
\hline \multicolumn{9}{|l|}{ VERBENACEAE } \\
\hline Aegiphila sp. & $\mathrm{X}$ & & & & & $\mathrm{X}$ & & \\
\hline \multicolumn{9}{|l|}{ VOCHYSIACEAE } \\
\hline Qualea grandiflora Mart. & & & & & & & & $\mathrm{X}$ \\
\hline TOTAL DE ESPÉCIES & 59 & 36 & 40 & 34 & 37 & 37 & 55 & 64 \\
\hline
\end{tabular}

Nesse sentido, a relação positiva observada entre a similaridade florística e a distância geográfica entre os fragmentos $\left(\mathrm{F}=50,200, \mathrm{p}=0,001, \mathrm{n}=28, \mathrm{r}^{2}=0,6588\right.$; Figura 2) demonstra que a proximidade entre fragmentos explica grande parte da variação observada na similaridade de espécies entre os fragmentos, reforçando a idéia de que fragmentos mais próximos, localizados dentro de unidades fisionômicas similares, possuem maior similaridade florística (OLIVEIRA FILHO e MACHADO, 1993).

Observou-se ainda uma relação marginalmente significativa entre o número de espécies por fragmento e a área dos fragmentos $(\mathrm{F}=2,462, \mathrm{p}=0,053, \mathrm{n}=8$, $\left.r^{2}=0,2911\right)$, indicando que fragmentos maiores devem apresentar maior número de espécies (Figura 3). Contudo, a variável independente explicou apenas $29 \%$ da riqueza 
de espécies nos fragmentos. Assim, é provável que outros fatores ecológicos, como a fitofisionomia local, o grau de antropização e a localização geográfica do fragmento, possam também afetar a riqueza de espécies nesses remanescentes florestais. Do mesmo modo, outros estudos também têm mostrado que a riqueza de espécies de remanescentes florestais é afetada pela área dos fragmentos (OUBORG, 1993; KOHN e WALSH, 1994), corroborando o padrão de riqueza de espécies em função da área dos fragmentos observado neste estudo. Primariamente, esse padrão decorre do próprio efeito da área, conforme predições da Teoria da Biogeografia Insular (MACARTHUR e WILSON, 1963). Assim, fragmentos maiores deveriam suportar maior número de espécies, especialmente porque são mais facilmente colonizados e as populações estão menos sujeitas a extinções locais (COX e MOORE, 1993). Além disso, o efeito de borda também deve ser considerado quando se avalia o efeito do tamanho do fragmento na riqueza de espécies. De modo geral, fragmentos menores sofrem maior efeito da borda, ocasionando maior perda de diversidade (MURCIA, 1995; METZGER, 1999).

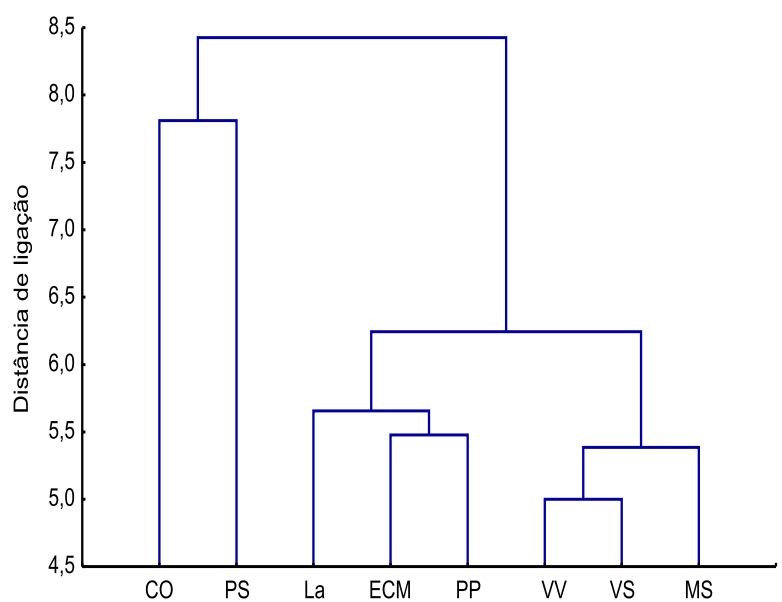

Figura 1 - Dendrograma de similaridade florística de oito fragmentos florestais no norte de Minas Gerais, Brasil (CO: COPASA; PS: Parque da Sapucaia; La: Lapinha; ECM: Escola Caio Martins; PP: Pedra Preta; VV: Vale Verde; VS: Volta da Serra; e MS: Mata da Serra).

Figure 1 - Cluster of floristic similarity for eight forest fragments in the north of Minas Gerais State, Brazil (CO: COPASA; PS: Parque da Sapucaia; La: Lapinha; ECM: Escola Caio Martins; PP: Pedra Preta; VV: Vale Verde; VS: Volta da Serra; MS: Mata da Serra).

R. Árvore, Viçosa-MG, v.31, n.1, p.135-144, 2007
Os resultados deste estudo evidenciam que a fitofisionomia local (fatores históricos) constitui importante elemento na florírica dos fragmentos florestais do semi-árido mineiro. No entanto, fatores ecológicos como as interações entre plantas e o ambiente físico contribuem para a composição de espécies nesses remanescentes. Além disso, o tamanho dos fragmentos constitui um indicador do número de espécies arbóreas nesses ambientes. Portanto, a fitofisionomia local e o tamanho dos fragmentos florestais devem ser considerados na elaboração de planos de manejo e preservação de espécies em fragmentos florestais no norte de Minas Gerais.

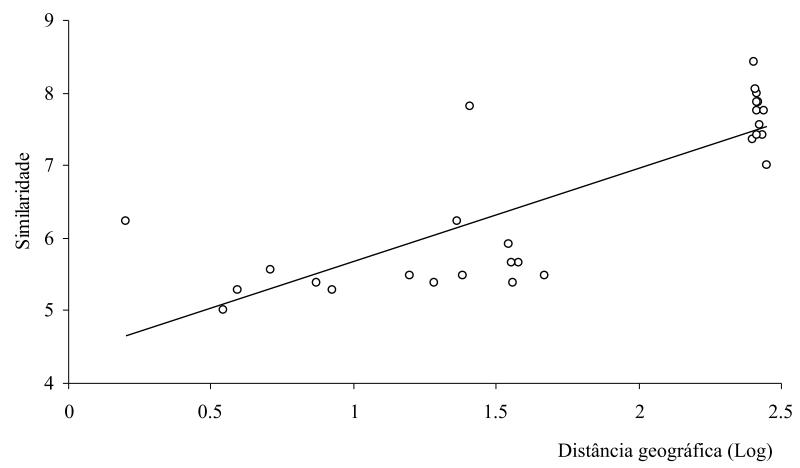

Figura 2 - Relação entre a distância de ligação (similaridade florística) e a distância geográfica linear de oito fragmentos florestais no norte de Minas Gerais, Brasil.

Figure 2-Relationship between the linkage distance (floristic similarity) and linear geographic distance of eight forest fragments in the north of Minas Gerais State, Brazil.

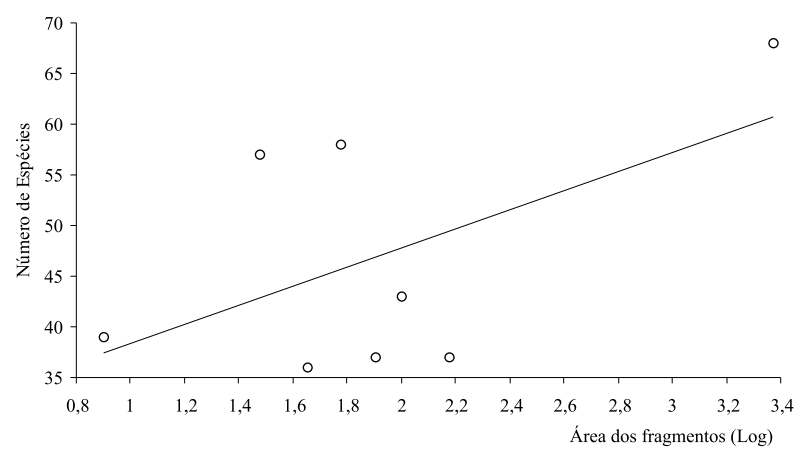

Figura 3 - Relação entre a área e a riqueza de árvores amostradas de oito fragmentos florestais no norte de Minas Gerais, Brasil.

Figure 3-Relationship between area and tree richness of eight forest fragments in the north of Minas Gerais State, Brazil. 


\section{AGRADECIMENTOS}

À FAPEMIG, pela concessão das Bolsas de Incentivo à Pesquisa e ao Desenvolvimento Tecnológico (BIPDT) para M. Fagundes e Y. R. F. Nunes e de Iniciação Científica para R. M. Santos (PROBIC - FAPEMIG/ UNIMONTES); aos Professores S. D'Angelo-Neto, pelo auxílio na identificação do material botânico, e B. G. Madeira, pela revisão do abstract e aos revisores anônimos deste artigo, pelas críticas e sugestões, contribuindo para a melhoria da sua versão final.

\section{REFERÊNCIAS}

ANGIOSPERM PHYLOGENY GROUP II. An update of the Phylogeny Group classification for the orders and families of flowering plants: APG II. Botanical Journal of the Linnaean Society, v.141, n.4, p.399-436, 2003.

BIERREGAARD, R.O.; DALE, V.H. Islands in an ever-changing sea: the ecological and socioeconomic dynamics of Amazonian rainforests fragments. In: SCHELLAS, J.; GREENBERG, R. (Ed.). Forest patches in tropical landscapes. Washington: University Island Press, 1996. p.187-204.

BRANDÃo, M. Área Mineira do Polígono das Secas / cobertura vegetal. Informe Agropecuário, v.17, n.181, p.5-9, 1994.

COX C.B.; MOORE, P.D. Biogeography: an ecological and evolutionary approach. 5. ed. Berlin: Blackwell Science, 1993. 326p.

ESPÍRITO SANTO, F.D.B. et al. Variáveis ambientais e a distribuição de espécies arbóreas em um remanescente de floresta estacional semidecídua montana no campus da Universidade Federal de Lavras, MG. Acta Botanica Brasilica, v.16, n.3, p.331-356, 2002.

FERNANDES, A. Fitogeografia Brasileira. Fortaleza: Multigraf, 1998. 205p.

FERNANDES, A. Biodiversidade da caatinga. In: ARAÚJO, E.L. et al. (Ed.). Biodiversidade, conservação e uso sustentável da flora do Brasil. Recife: UFRPE e SBB, 2002. p.42-43.
KELLMAN, M.; TACKABERRY, R.; MEAVE, J. The consequences of prolonged fragmentation: lessons from tropical gallery forests. In: SCHELLAS, J.; GREENBERG, R. (Ed.). Forest patches in tropical landscapes. Washington: University Island Press, 1996. p.37-58

KOHN, D.D.; WALSH, D.M. Plant species richness: the effect of island size and habitat diversity. Journal of Ecology, v. 82, p.367-377, 1994.

LUDWIG, J.A.; REYNOLDS, J.F. Statistical ecology: a primer on methods and computing. New York: John Wiley \& Sons, 1996.337p.

MACARTHUR, R.H.; WILSON, E.O. An equilibrium theory of island biogeography. Evolution, v.17, p.373-387, 1963.

MARTIUS, C.F.P. A fisionomia do reino vegetal no Brasil. Anuário Brasileiro de Economia Florestal, v.10, p.209-227, 1958.

MELLO-BARRETO, H.L. Regiões fitogeográficas de Minas Gerais. Boletim Geográfico, v.14, p.14-28, 1942.

METZGER, J.P. Estrutura da paisagem e fragmentação: análise bibliográfica. Anais da Academia Brasileira de Ciências, v.71, p.445-463, 1999.

MEYER, S.T. et al. Composição florística da vegetação arbórea de um trecho de floresta galeria do Parque Estadual do Rola-Moça na região metropolitana de Belo Horizonte, $\mathrm{MG}$, Brasil. Acta Botanica Brasilica, v.18, n.4, p.701-709, 2004.

MURCIA, C. Edge effects in fragmented forest: implications for conservation. Trends in Ecology and Evolution, v.10, n.1, p.58-62, 1995.

NASCIMENTO, A.R.T.; FELFILI, J.M.; MEIRELLES, E.M. Florística e estrutura da comunidade arbórea de um remanescente de floresta estacional decidual de encosta, Monte Alegre, GO, Brasil. Acta Botanica Brasilica, v.18, n.3, p.659-669, 2004.

R. Árvore, Viçosa-MG, v.31, n.1, p.135-144, 2007 
NUNES, Y.R.F. et al. Variações da fisionomia, diversidade e composição de guildas da comunidade arbórea em um fragmento de Floresta Semidecidual em Lavras, MG. Acta Botanica Brasilica, v.17, n.2, p.213-229, 2003.

OLIVEIRA FILHO, A.T. et al. Effects of canopy gaps, topography, and soils on the distribution of woody species in a central Brazilian deciduous dry forest. Biotropica, v.30, n.3, p.362-375, 1998.

OLIVEIRA FILHO, A.T.; MACHADO, J.N.M. Composição florística de uma floresta semidecídua montana na serra de São José, Tiradentes, Minas Gerais. Acta Botanica Brasilica, v.7, n.2, p.71-88, 1993.

OUBORG, N.J. Isolation, population size and extinction: the classical and meta population approaches applied to vascular plants along the Dutch Rhine-system. Oikos, v.66, p.298-308, 1993.

PEDRALLI, G. Florestas secas sobre afloramentos de calcário em Minas Gerais: florística e fisionomia. Bios, v.5, n.5, p.81-88, 1997.

RIBAS, R.F. et al. Composição florística de dois trechos em diferentes etapas serais de uma Floresta Estacional Semidecidual em Viçosa, Minas Gerais. Revista Árvore, v.27, n.6, p.821-830, 2003.

R. Árvore, Viçosa-MG, v.31, n.1, p.135-144, 2007
SOUZA, J.S. et al. Análise das variações florísticas e estruturais da comunidade arbórea de um fragmento de floresta semidecídua às margens do rio Capivari, Lavras-MG. Revista Árvore, v.27, n.2, p.185-206, 2003.

van den BERG, E.; OLIVEIRA FILHO, A.T. Spatial partitioning among tree species within an area of tropical montane gallery forest in south-eastern Brazil. Flora, v.194, p.249-266, 1999.

VELOSO, H.P.; RANGEL FILHO, A.L.R.; LIMA, J.C.A. Classificação da vegetação brasileira adaptada a um sistema universal. Rio de Janeiro: IBGE,

Departamento de Recursos Naturais e Estudos Ambientais, 1991. 123p.

VIANA, V.M.; PINHEIRO, L.A.F.V.

Conservação da biodiversidade em

fragmentos florestais. Série Técnica IPEF, v. 12, n.32, p. 25-42, 1998.

WILLIAMS-LINERA, G.; DOMÍNGUEZ-GASTELÚ, V.; GARCÍA-ZURITA, M.E. Microenvironment and floristics of different edges in a fragmented tropical rainforest. Conservation Biology, v.12, n.5, p.1091-1102, 1998.

ZAR, J.H. Biostatistical Analysis. 3. ed. New Jersey: Prentice Hall, 1996. 662p. 